\title{
A colourimetric method for the determination of the degree of chemical cross-linking in aspartic acid-based polymer gels
}

\author{
B. Gyarmati ${ }^{1}$, N. Hegyesi ${ }^{1,2}$, B. Pukánszky ${ }^{1,2}$, A. Szilágyi ${ }^{*}$ \\ ${ }^{1}$ Department of Physical Chemistry and Materials Science, Budapest University of Technology and Economics, Budafoki \\ út 8., H-1111 Budapest, Hungary \\ ${ }^{2}$ Institute of Materials Science and Environmental Chemistry, Research Centre for Natural Sciences, Hungarian Academy \\ of Sciences, P.O. Box 286, H-1519 Budapest, Hungary
}

Received 9 May 2014; accepted in revised form 5 October 2014

\begin{abstract}
A 2,4,6-trinitrobenzenesulphonic acid (TNBS)-based assay is developed to determine the degree of chemical cross-linking in aspartic acid-based polymer gels. The conventional colourimetric method for the quantitative determination of amine groups is difficult to use in polymer networks; thus, an improved method is developed to analyse polymer gels swollen in dimethyl sulfoxide (DMSO). Reaction products of the derivatizing reaction are examined by NMR. The chemical stability of the reagent is increased in DMSO, and the method shows satisfactory linearity and accuracy. The degree of chemical cross-linking in the investigated gels is close to its theoretical maximum, but the conversion of the pendant amine groups to cross-linking points is strongly dependent on the feed composition of the gels.
\end{abstract}

Keywords: polymer gels, 2,4,6-trinitrobenzene-sulphonic acid, poly(aspartic acid), degree of cross-linking, cross-linking ratio

\section{Introduction}

Polymer hydrogels are good candidates for several bio-related applications because of their large waterabsorbing capacity, soft tissue-like properties, viscoelastic character and permeability to the influx and efflux of small molecular compounds [1-5]. The swelling properties and mechanical strength of the hydrogels are determined mainly by the cross-link density, which is the number of physical and chemical net points that connect polymer chains in a unit volume of gel [1, 6-8]. Although several theoretical models are available for the estimation of crosslinking density (e.g., Flory-Rehner swelling theory [9]), these models have several limitations and cannot distinguish chemical from physical net points. However, chemical net points are of particular importance because they are stable under physiological conditions and determine the maximum degree of swelling of hydrogels.
The goal of this work was to develop a simple analytical method for quantifying the number of chemical cross-links in aspartic acid-based polymer gels [10-13]. Polysuccinimide (PSI) gels are obtained by cross-linking PSI with diamines, and cross-linked PSI gels can be hydrolysed into poly(aspartic acid) (PASP) gels. During the cross-linking of PSI, some of the diamine molecules remain unreacted, whereas others react with the polymer through only one of their amine groups so pendant amine groups remain in the network. Cross-linker molecules reacting with both amine groups form chemical cross-links between polymer chains. The cross-linking ratio $\left(X_{\text {FEED }}\right)$ is defined as the number of moles of crosslinker molecules per the number of moles of repeating units of the polymer [14]. The structure of the formed network can be characterized by the degree of chemical cross-linking which is the molar ratio of diamine molecules that form chemical cross-link-

\footnotetext{
*Corresponding author, e-mail: aszilagyi@mail.bme.hu (c) BME-PT
} 
ing points to the repeating units of the polymer in PSI and PASP gels. Essentially, the degree of chemical cross-linking is only equal to the cross-linking ratio if the conversion rate of the reaction is $100 \%$. The degree of chemical cross-linking of PASP gels was previously determined by Torma et al. [14]. The concentration of unreacted cross-linker molecules was quantified using ninhydrin, whereas the concentration of pendant amine groups was determined by aqueous acid-base potentiometric titration. However, the equilibration time can be long for every titration step in the case of cross-linked polymers. Thus, this method is very time-consuming because a large number of data points must be determined to obtain a reliable titration curve. A method that requires considerably fewer data points would be highly beneficial.

In the colourimetric method developed in this study, the degree of chemical cross-linking was determined using 2,4,6-trinitrobenzene-sulphonic acid (TNBS), which is a well-known reagent for the quantitative determination of primary amine groups in chromatography. In alkaline aqueous solution, TNBS reacts with amine groups via bimolecular aromatic nucleophilic substitution, resulting in a yellow adduct with absorption maxima at $340 \mathrm{~nm}$ (trinitrophenyl amine) and $420 \mathrm{~nm}$ (Meisenheimer complex) [15-20]. Simultaneously, TNBS hydrolyses to picric acid, which exhibits overlapping peaks with the main product, trinitrophenyl amine (Figure 1) [21]. The reaction constant of the hydrolysis is a few orders of magnitude smaller than that of the main reaction (e.g., the pseudo first-order rate constant for the amine group of lysine is $9 \cdot 10^{-4} 1 / \mathrm{s}$ whereas for hydroxyl groups is $1.4 \cdot 10^{-7} 1 / \mathrm{s}$ ) [22]; thus, the side reaction does not interfere with the quantitative determination of small molecules [15, 22]. However, the side reaction is not negligible in slow reactions; hence, the conventional TNBS assay cannot be applied directly to determine the concentration of amine groups in polymer gels. Moreover, the rate of the hydrolysis to picric acid strongly depends on the ionic strength [22], which is difficult to maintain at a well-defined value in the presence of a polyelectrolyte gel $[2,8,23]$. The dissimilar reaction rates of by-product formation in the analyte and in the blank solution might cause inaccuracies during absorbance measurements and in the quantitative determination of amine groups. Some researchers tried to solve this difficulty by hydrolysing the gels to small molecules in strongly acidic solution after the reaction with TNBS [24-28]. However, controlling the degradation of the polymer might be difficult under the extreme reaction conditions, which resulting in the formation of poorly defined oligomers and other by-products. Consequently, we employed a new strategy to exclude the formation of picric acid by changing the solvent of the assay to DMSO and to be able to perform the assay under mild reaction conditions. The reaction mechanism is proposed based on ${ }^{1} \mathrm{H}$ NMR measurements of the model reaction of TNBS and the cross-linker molecules. The degree of chemical cross-linking was determined as a function of the feed composition of the gels.

\section{Experimental}

\subsection{Materials and instruments}

Imidazole (puriss p.a.), 1,4-diaminobutane (DAB, 99\%) and 2,4,6-trinitrobenzenesulphonic acid (TNBS, $1 \mathrm{M}$ in $\mathrm{H}_{2} \mathrm{O}$, analytical reagent) solutions were acquired from Sigma-Aldrich Ltd., Hungary. Dibutylamine (DBA, 99\%), sodium tetraborate decahy-

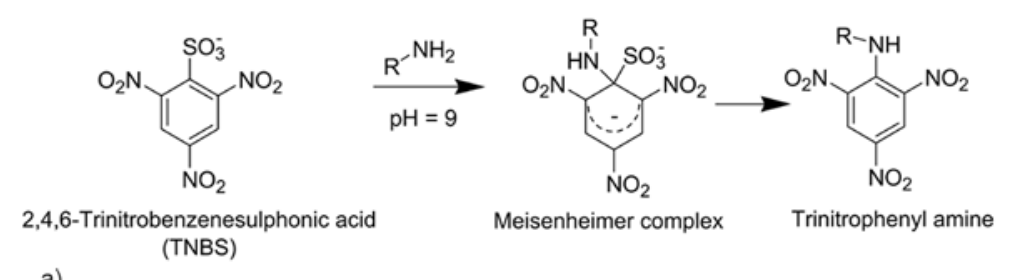

a)

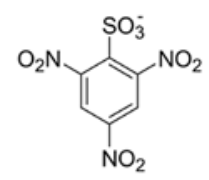

b)

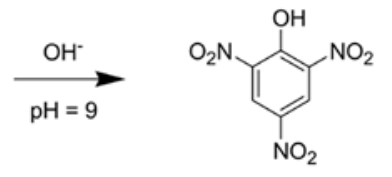

Picric acid (by-product)

Figure 1. Reaction of 2,4,6-trinitrobenzenesulphonic acid (TNBS) with a) primary amines and b) hydroxyl ions 
drate (a.r.), cysteamine hydrochloride (97\%), cystamine dihydrochloride $(98 \%)$, potassium chloride (99.5\%), sodium bromate (99\%), poly(vinyl alcohol) (PVA, $M_{\mathrm{W}}=60 \mathrm{kDa}, 98 \%$ hydrolysed), glutardialdehyde (GDA, 25wt\% solution in water for synthesis), S-aspartic acid (99\%) and dithiothreitol (DTT, for biochem.) were purchased from Merck Ltd., Hungary. N,N-Dimethylformamide (DMF, pure) was bought from Lach Ner, Czech Republic. Dimethyl sulphoxide (DMSO, analytical grade) was obtained from Fisher Chemical, Hungary. De-ionized water (Milli-Q reagent grade, $\kappa>18.2 \Omega \cdot \mathrm{m}$, Millipore, USA) was used for each analysis and for the preparation of buffer solutions. All reagents and solvents were used without further purification.

The $\mathrm{pH}$ of the buffer solutions was verified using a pH/ion analyser (Radelkis OP-271/1, Hungary). Absorbance measurements were performed using a Specord 200 UV-Vis spectrophotometer (Analytik Jena, Germany) with a quartz cuvette (path length $=$
$1 \mathrm{~cm}, V=3 \mathrm{~mL})$. The spectral resolution was $1 \mathrm{~nm}$, and the scanning speed was $10 \mathrm{~nm} / \mathrm{s}$. Nuclear magnetic resonance (NMR) experiments were performed on a Bruker Avance 300 spectrometer (USA) operating at $300 \mathrm{MHz} .{ }^{1} \mathrm{H}$ NMR spectra were recorded using 128 scans.

A buffer solution of $\mathrm{pH}=8$ was prepared from imidazole $(c=0.1 \mathrm{M})$; the $\mathrm{pH}$ was adjusted to 8 by the addition of $1 \mathrm{M} \mathrm{HCl}$. A buffer solution of $\mathrm{pH}=9.3$ was prepared from sodium tetraborate decahydrate $(c=0.1 \mathrm{M})$ and used in aqueous reactions of TNBS. The ionic strength of the buffers was maintained at $0.15 \mathrm{M}$ by the addition of $\mathrm{KCl}$.

\subsection{Synthesis}

Polysuccinimide (PSI) was synthesized by the thermal polycondensation of aspartic acid as reported in a previous study [29]. The average molecular weight of PSI was $M_{\mathrm{W}}=56 \mathrm{kDa}$. PSI-DAB gels were prepared by cross-linking PSI with DAB (Figure 2). In
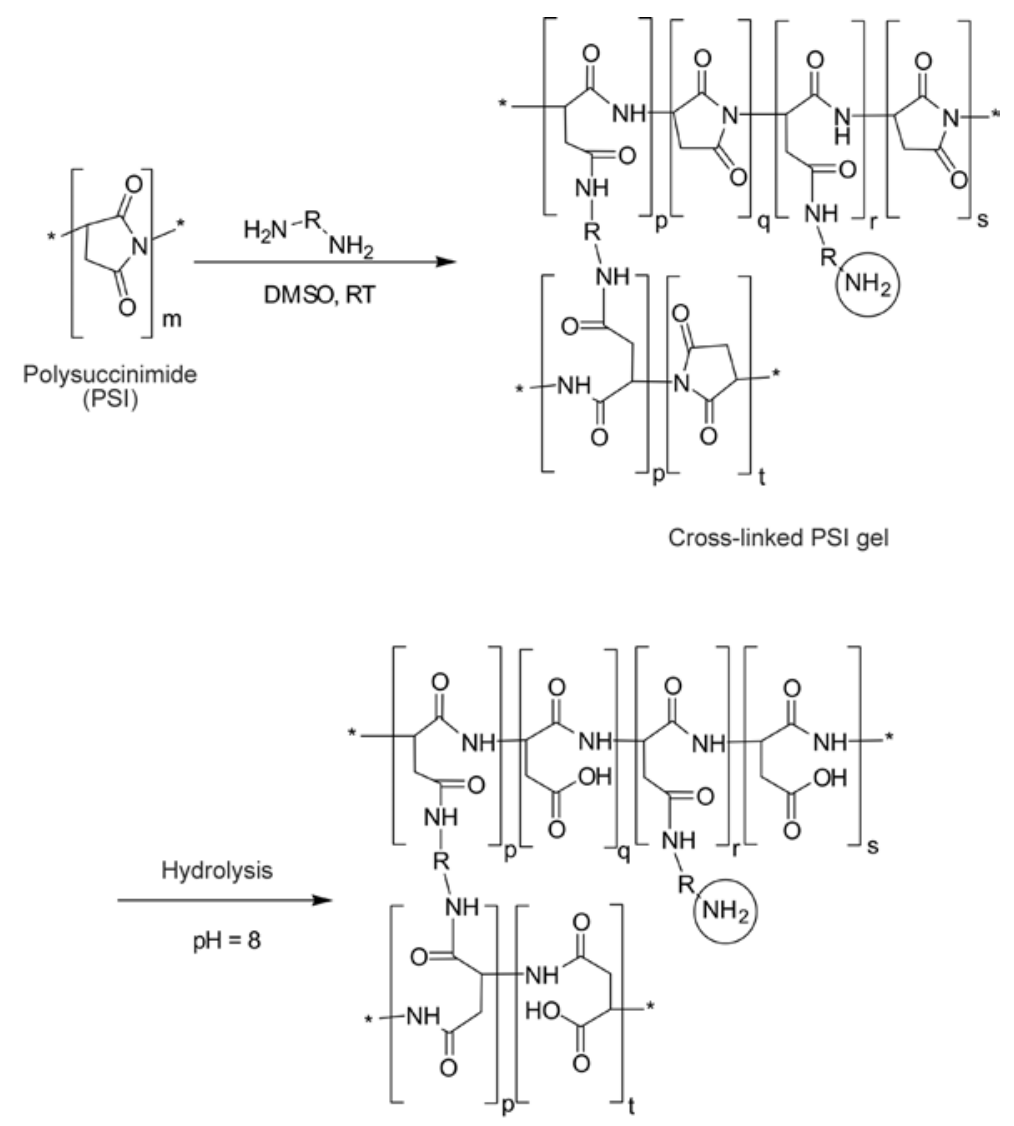

Cross-linked poly(aspartic acid) (PASP) gel

$$
\mathrm{H}_{2} \mathrm{~N}^{-\mathrm{R}} \mathrm{NH}_{2}=\mathrm{H}_{2} \mathrm{~N} \mathrm{NH}_{2} \quad \text { 1,4-diaminobutane (DAB) }
$$

Figure 2. The synthesis of polysuccinimide (PSI) and poly(aspartic acid) (PASP) gels 
brief, PSI was dissolved in DMSO (9.70 wt $\%)$, and the solution of DAB ( $8.80 \mathrm{wt} \%$ in DMSO) was added dropwise to the polymer solution. The precursor solution of gels was transferred into Eppendorf tubes $(V \cong 1 \mathrm{~mL})$. Although the gelation of the solution could be visually observed after a few minutes (Table 1), the gels were kept in the tubes for 24 hours to complete the cross-linking process. The

Table 1. Cross-linking ratio, gelation time and degree of swelling of the gels

\begin{tabular}{|c|c|c|c|c|}
\hline Sample & $\begin{array}{c}\mathbf{X}_{\text {FEED }^{\mathbf{a}}} \\
{[-]}\end{array}$ & $\begin{array}{c}\mathbf{t}_{\text {gel }}{ }^{\mathbf{b}} \\
{[\mathbf{m i n}]}\end{array}$ & $\begin{array}{c}\mathbf{Q}_{\mathbf{m}}{ }^{\mathbf{c}} \\
{[-]}\end{array}$ & $\begin{array}{c}\mathbf{Q}_{\mathbf{m}, \mathbf{p H}=\mathbf{8}^{\mathbf{d}}} \\
{[-]}\end{array}$ \\
\hline $\mathrm{A}$ & $1 / 5$ & $3-5$ & 5.0 & 7.5 \\
\hline $\mathrm{B}$ & $1 / 10$ & $5-8$ & 7.2 & 15.2 \\
\hline $\mathrm{C}$ & $1 / 15$ & $8-10$ & 9.0 & 18.3 \\
\hline $\mathrm{D}$ & $1 / 20$ & $18-20$ & 9.3 & 20.5 \\
\hline
\end{tabular}

${ }^{\mathrm{a}} X_{\mathrm{FEED}}$ : cross-linking ratio which is the number of moles of crosslinker molecules per the number of moles of repeating units of the polymer,

${ }^{\mathrm{b}} t_{\mathrm{gel}}$ : gelation time of PSI-DAB gel determined by visual observation,

${ }^{\mathrm{c}} Q_{\mathrm{m}}$ : the mass ratio of the prepared PSI-DAB gel to the dried gel,

${ }^{\mathrm{d}} Q_{\mathrm{m}, \mathrm{pH}=8}$ : the mass ratio of the PASP-DAB gel at $\mathrm{pH}=8$ to the dried gel
PSI-DAB gels were immersed in aqueous buffer solution $(\mathrm{pH}=8)$ and the gels became opaque at first because water is a poor solvent of PSI. The PSI-DAB gels hydrolysed to PASP-DAB gels within 3 days and homogeneous, transparent gels were yielded. The degree of swelling $\left(Q_{\mathrm{m}}\right)$ of the PSI-DAB gel is defined as the mass ratio of the prepared gel to the dried gel; $Q_{\mathrm{m}, \mathrm{pH}=8}$ of the PASP-DAB gel is defined as the mass ratio of PASP-DAB gel swollen at $\mathrm{pH}=8$ to the dried gel. The mass of the dried gel was determined by immersing the gel piece into water, followed by a drying step in vacuum at $25^{\circ} \mathrm{C}$.

\subsection{Determination of degree of chemical cross-linking of PASP-DAB hydrogels in water}

The degree of chemical cross-linking of PASP-DAB gels was determined in two main steps as shown in Figure 3. In the first step, unreacted diamine molecules were extracted from the PASP-DAB gels ( $m \cong 1 \mathrm{~g}$ ) by washing the gels with buffer solution $(5 \cdot 1000 \mu \mathrm{L}, \mathrm{pH}=9.3)$. The amine content of the

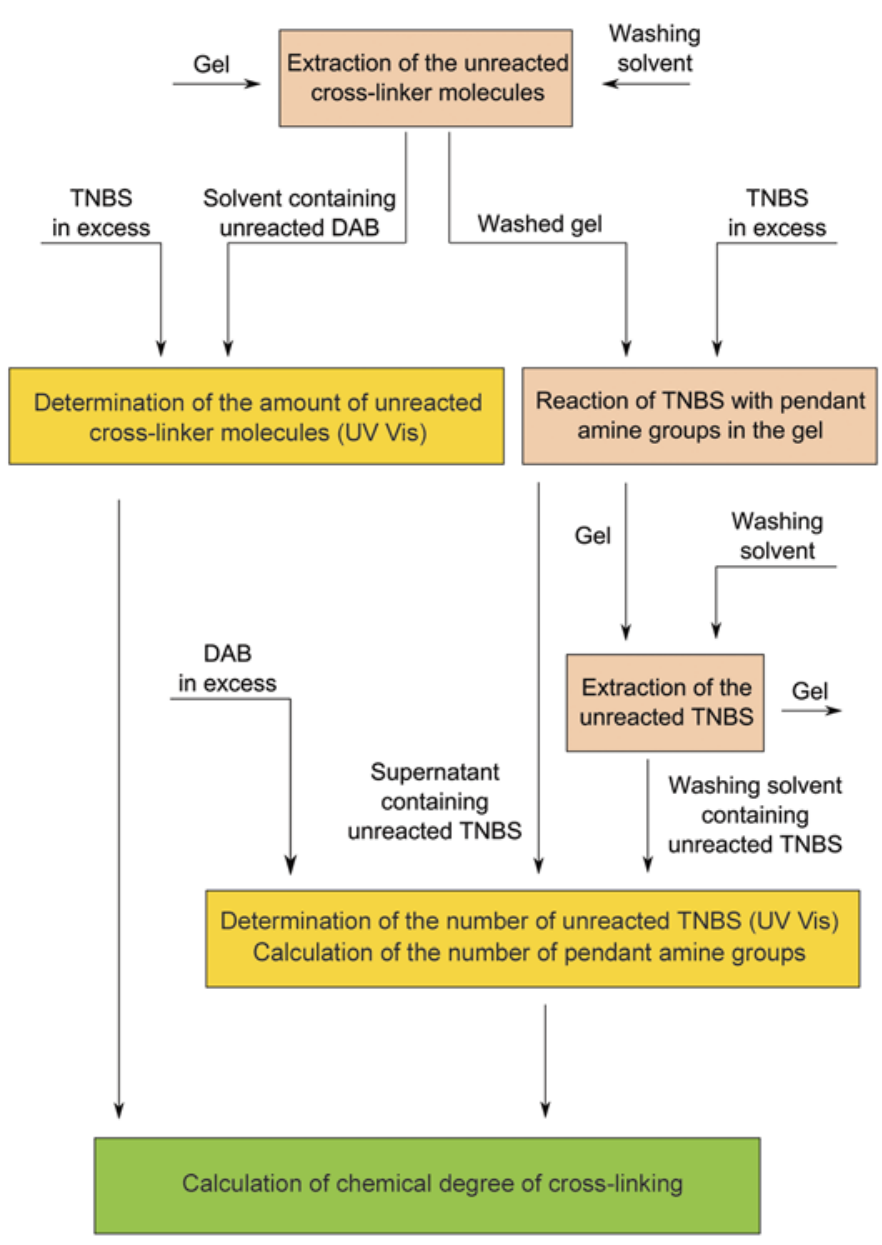

Figure 3. Flow-chart of the quantification of unreacted cross-linker molecules and pendant amine groups inside the gels 
washing solution was determined after adding TNBS ( $50 \mu \mathrm{L}, 30 \mathrm{mM}$ TNBS in water) in excess to $2000 \mu \mathrm{L}$ of the analyte. The absorbance of the analyte solution was measured after 30 min against a blank buffer solution $(\mathrm{pH}=9.3)$ containing TNBS at the same concentration as in the analyte. Calibration of the method was done using $\mathrm{DAB}$ in alkaline aqueous solution $\left(c_{\mathrm{TNBS}}=7.3 \cdot 10^{-4} \mathrm{M}, \mathrm{pH}=9.3, \lambda=420 \mathrm{~nm}\right.$, $\left.\varepsilon=15364 \mathrm{M}^{-1} \cdot \mathrm{cm}^{-1}, r^{2}=0.9956\right)$.

In the second step, a washed gel sample ( $m \cong 0.01 \mathrm{~g}$ ) was immersed in aqueous solution of TNBS $(500 \mu \mathrm{L}$, $3 \mathrm{mM}, \mathrm{pH}=9.3$ ) and reacted for $3 \mathrm{~h}$ at $25^{\circ} \mathrm{C}$. The gel was removed and the unreacted TNBS was extracted from the gel by washing with buffer solution (3.500 $\mu \mathrm{L}, \mathrm{pH}=9.3)$. The TNBS content of the solution was determined by the addition of DAB $(50 \mu \mathrm{L}$, $8.80 \mathrm{wt} \%$ ) in excess to $5000 \mu \mathrm{L}$ of the analyte. The concentration of unreacted reagent was determined from its absorbance spectrum.

\subsection{Determination of degree of chemical cross-linking of PSI-DAB gels in DMSO}

The degree of chemical cross-linking of PSI-DAB gels was determined similarly as in the case of PASP$\mathrm{DAB}$ gels, but the solvent was replaced from water to DMSO (Table 1). Thus, in the first step (Figure 3), unreacted diamine molecules were removed from the PASP-DAB gels ( $m \cong 1 \mathrm{~g}$ ) by washing the gels with DMSO (5.1000 $\mu \mathrm{L}$ DMSO). The amine content of the washing solution was determined after adding TNBS ( $50 \mu \mathrm{L}, 300 \mathrm{mM}$ in DMSO) to $2000 \mu \mathrm{L}$ of the analyte. The absorbance of the analyte solution was measured after 30 min against the blank solution of TNBS in DMSO. Calibration was done in DMSO

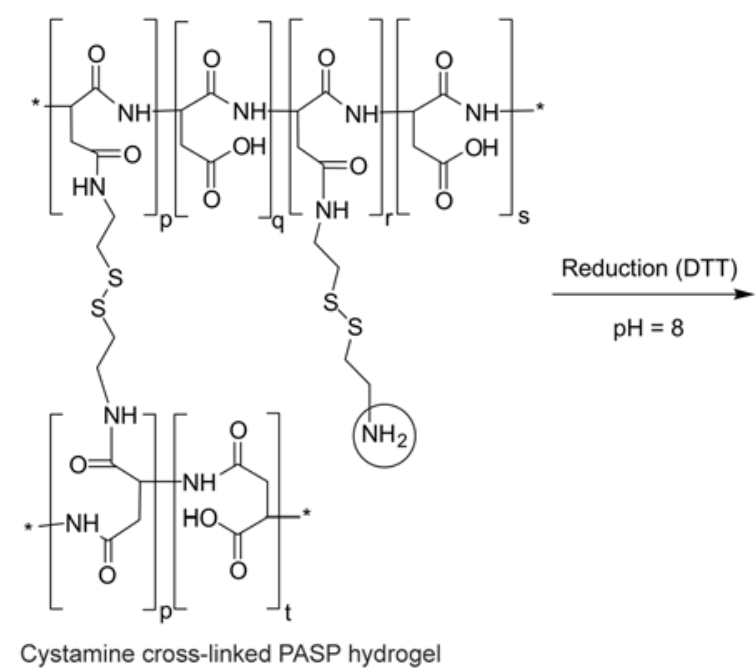

using TNBS in excess $\left(c_{\mathrm{TNBS}}=7.3 \cdot 10^{-3} \mathrm{M}, \lambda=\right.$ $\left.437 \mathrm{~nm}, \varepsilon=625 \mathrm{M}^{-1} \cdot \mathrm{cm}^{-1}, r^{2}=0.9992\right)$.

In the second step, a washed gel sample ( $m \cong 0.01 \mathrm{~g})$ was immersed in TNBS ( $375 \mu \mathrm{L}, 30 \mathrm{mM}$ in DMSO) and reacted for 1 day. The gel was removed and the unreacted TNBS was extracted from the gel by washing (3.500 $\mu \mathrm{L}$ DMSO). The TNBS content of the solution was determined by the addition of DAB (125 $\mu \mathrm{L}, 8.80 \mathrm{wt} \%$ in DMSO) in excess to $5000 \mu \mathrm{L}$ of the analyte. The concentration of the reaction product was determined from its absorbance spectrum. Calibration was done in DAB excess $\left(c_{\mathrm{DAB}}=\right.$ $25 \cdot 10^{-3} \mathrm{M}, \lambda=437 \mathrm{~nm}, \varepsilon=18393 \mathrm{M}^{-1} \cdot \mathrm{cm}^{-1}, r^{2}=$ 0.9995).

\subsection{Accuracy and stability}

A widely accepted reference method does not exist for the determination of the number of pendant amine groups. Thus, we applied a method based on the determination of small molecules to determine accuracy because the TNBS assay for small molecular amines is well-established. Disulphide crosslinked PSI gels (PSI-CYS) were synthesized by the reaction of PSI and cystamine (CYS) (Figure 2). $950 \mathrm{mg}$ of polymer solution (PSI in DMF, $5.00 \mathrm{wt} \%$ ) was diluted with $451 \mathrm{mg}$ DMF, and then $23 \mathrm{mg}$ cystamine hydrochloride was dissolved in the same solution. $34 \mu \mathrm{L}$ dibutylamine as a deprotonating agent was added dropwise to the solution under continuous stirring. The solution was poured into Eppendorf tubes $(V \cong 1 \mathrm{~mL}$ ) and kept there for $24 \mathrm{~h}$ to yield gels. The concentration of pendant amine groups in PSI-CYS gel was determined in DMSO as described previously in the case of PSI-DAB gels after careful

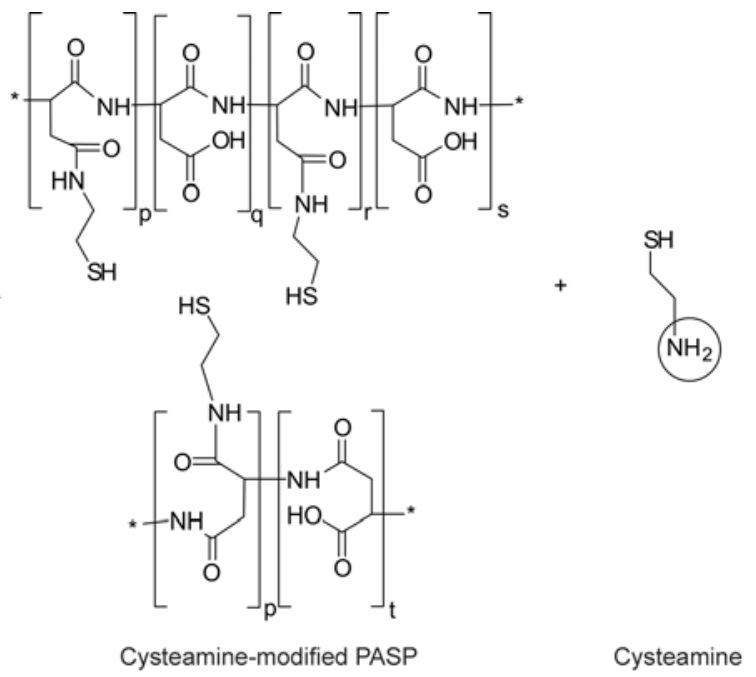

Figure 4. Reduction of cystamine cross-linked poly(aspartic acid) (PASP) gels to cysteamine-modified PASP and cysteamine 
removal of cystamine by washing with $0.1 \mathrm{M} \mathrm{HCl}$. For the reference measurements, the PSI-CYS gels were hydrolysed to disulphide cross-linked PASP (PASP-CYS) gels. A given amount $(m \cong 0.1 \mathrm{~g}$ ) of PASP-CYS gel was placed into a dialysis tube (cutoff molecular weight was $12 \mathrm{kDa}$ ) filled with $10 \mathrm{ml}$ of a buffer solution $(\mathrm{pH}=8)$ containing $10 \mathrm{mM}$ DTT to reduce the disulphide bonds inside the hydrogel (Figure 4). Solutions of the reduced polymer and cysteamine were dialysed against water. The cysteamine concentration in the outer solution was determined with TNBS in the presence of sodium bromate $\left(c=10^{-4} \mathrm{M}\right)$. Calibration was done using cysteamine in aqueous buffer solution over the concentration range of $1.0 \cdot 10^{-5}$ to $8.0 \cdot 10^{-5} \mathrm{M}\left(c_{\mathrm{TNBS}}=\right.$ $7.3 \cdot 10^{-4} \mathrm{M}, \lambda=420 \mathrm{~nm}, \varepsilon=11727 \mathrm{M}^{-1} \cdot \mathrm{cm}^{-1}$, $\left.r^{2}=0.9998\right)$.

The stability of TNBS was determined in DMSO. Chemically cross-linked poly(vinyl alcohol) (PVA) gels were synthesized as it follows. $3.0 \mathrm{~g}$ of PVA solution (12 wt\%) was diluted with $1.7 \mathrm{~g}$ of deionised water. Subsequently, $150 \mathrm{mg}$ of $1 \mathrm{M}$ GDA as a crosslinker and $100 \mu \mathrm{L}$ of $20 \% \mathrm{HCl}$ as a catalyst were added to the solution of PVA under continuous stirring at $25^{\circ} \mathrm{C}$. The pre-cursor solution was transferred into Eppendorf tubes and kept there for $24 \mathrm{~h}$. Unreacted cross-linker molecules were removed from the PVA gels by careful washing with de-ionized water. Lastly, the PVA hydrogels were immersed in DMSO, and the solvent was changed several times to produce PVA gels swollen in DMSO. A sample of PVA gel ( $m \cong 0.1 \mathrm{~g})$ was immersed in TNBS ( $300 \mu \mathrm{L}, 5 \mathrm{mM}$ in DMSO) and incubated for 3 days.

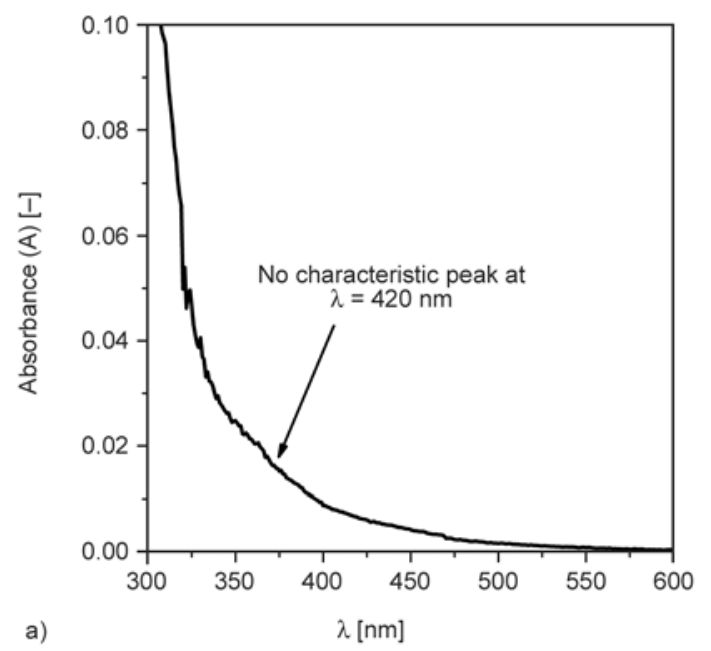

The gel was washed with DMSO $(3 \cdot 500 \mu \mathrm{L})$ to extract TNBS. The collected solution of TNBS was diluted with DMSO to $5000 \mu \mathrm{L}$. $50 \mu \mathrm{L}$ DAB (8.80 wt $\%$ in DMSO) was added to $2000 \mu \mathrm{L}$ of the diluted solution of TNBS. The absorbance spectrum of the solution was measured after $30 \mathrm{~min}$ against DMSO containing DAB at the same concentration as in the analyte.

\section{Results and discussion \\ 3.1. Determination of degree of chemical cross-linking in water}

The unreacted DAB molecules were extracted from the PSI-DAB gel and reacted with TNBS at $\mathrm{pH}=9.3$. Figure $5 \mathrm{a}$ shows the absorption spectrum of the solution after 30 min (sample D in Table 1). The characteristic peak at $420 \mathrm{~nm}$ could not be detected. Thus, the concentration of cross-linker molecules is below the detection limit of the method $\left(1.2 \cdot 10^{-5} \mathrm{M}\right)$ which means that less than $0.1 \%$ of the DAB molecules remain unreacted after the cross-linking process independently of the cross-linking ratio. Thus, each cross-linker molecule is attached to the polymer chain through at least one of its functional groups, in agreement with previously reported results [14]. After the immersion of the PASP-DAB gel into TNBS, the solution became yellow within 3 hours. A broad peak could be identified at $357 \mathrm{~nm}$ in the absorbance spectrum of the solution (Figure $5 b$ ). Because the supernatant must not contain amine groups, we can assume that the yellow compound is the hydrolysed by-product of TNBS, picric acid. The side reaction might have a larger reaction rate

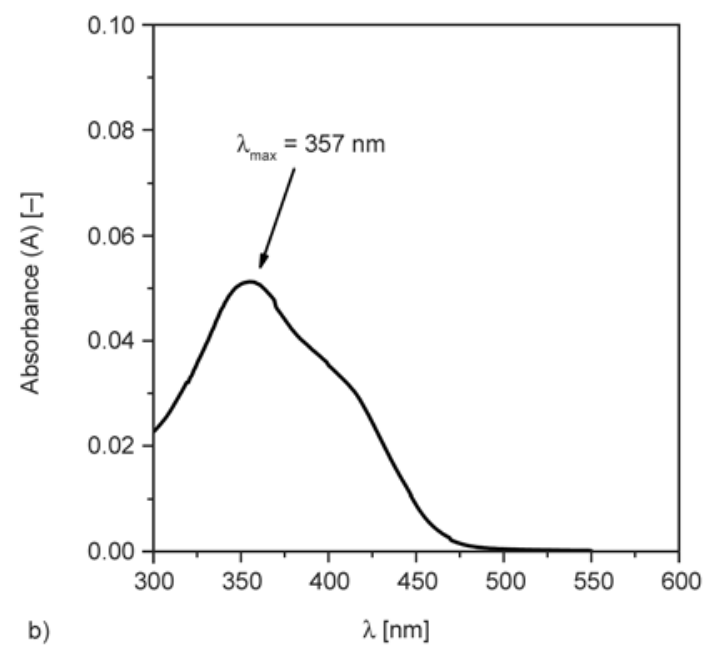

Figure 5. a) Absorption spectrum of the extracted swelling solution of PASP-DAB gel after the addition of TNBS in excess in aqueous buffer $(\mathrm{pH}=9.3)$; $\mathrm{b}$ ) absorption spectrum of the supernatant of PASP-DAB gels after $3 \mathrm{~h}$ incubation with TNBS in aqueous solution $(\mathrm{pH}=9.3)$ 
in the presence of the PASP gel, as indicated by the absence of a yellow product in the blank solution after the same reaction time. The dissimilar reaction rate of hydrolysis of TNBS can be explained by the difference in ionic strengths, i.e., PASP polyelectrolyte gels carry mobile counter-ions, which have a poorly defined effect on reaction kinetics. As a consequence, the number of pendant amines cannot be determined in aqueous solution and the reaction medium must be replaced to exclude side-reactions.

\subsection{Reaction mechanism in DMSO}

TNBS reacts with amine groups in DMSO as well, but several other factors must be considered. Contrary to the protic character of water, DMSO is a polar aprotic solvent. TNBS is a strong acid and exists in its dissociated form in alkaline aqueous solutions. Its dissociation is hindered in DMSO. Thus, TNBS remains protonated before the reaction with amine groups, which affects the mechanism of adduct formation. The stability of the Meisenheimer complex might also be different in DMSO [30]. We carried out a model reaction between TNBS and 1,4-diaminobutane in $\mathrm{d}_{6}$-DMSO to propose the possible reaction mechanism. We confine our discussion to two cases that are relevant to the present analysis: first, a large excess of TNBS was maintained, and second, a large excess of DAB was maintained (the molar ratios of TNBS to DAB were 3 and $1 / 3$, respectively). ${ }^{1} \mathrm{H}$ NMR spectra were recorded after 8 hours of reaction time. A detailed analysis of the reaction mechanism lies beyond the scope of the present study, and only the main conclusions are drawn here. The results of a detailed NMR analysis are to be published in another paper.

In all probability, sulphonic acid and primary amine groups form a salt in the dipolar solvent in a large excess of TNBS (Figure 6, I), followed by the nucleophilic addition of the primary amine group onto TNBS (Figure 6, II). The primary amine groups in the analyte can act as a base catalyst in the elimination of sulphonic acid groups (Figure 6, III). Accordingly, the conversion of TNBS to its amine-substituted derivative requires an excess of primary amine groups. Lastly, nucleophilic attack at the unsubstituted ring positions of TNBS may also occur in the large excess of primary amine groups (Figure 6, IV) $[20,30]$.

In a large excess of TNBS, the peak of the unreacted reagent appears at $8.8 \mathrm{ppm}(\mathrm{H}-\mathrm{a}$ nuclei) in Figure 7. A relatively small peak at $8.9 \mathrm{ppm}$ indicates the presence of the aniline derivative (Figure 6, III, H-b nuclei), assuming that a reaction time of 8 hours is sufficient for the total conversion of the Meisenheimer adduct (Figure 6, II) to the corresponding aniline (Figure 6, III). The broad peak at $7.6 \mathrm{ppm}$ can be assigned to the salt of TNBS and DAB (Figure $6, \mathrm{I})$. The assumption is supported by the intensity integrals (I) of the peaks: $\mathrm{I}(\mathrm{H}-\mathrm{c}): \mathrm{I}(\mathrm{H}-\mathrm{d}): \mathrm{I}(\mathrm{H}-\mathrm{e})=$ $3: 2: 2$. The integrals indicate that both amine groups of $\mathrm{DAB}$ are protonated as shown in Figure 6.

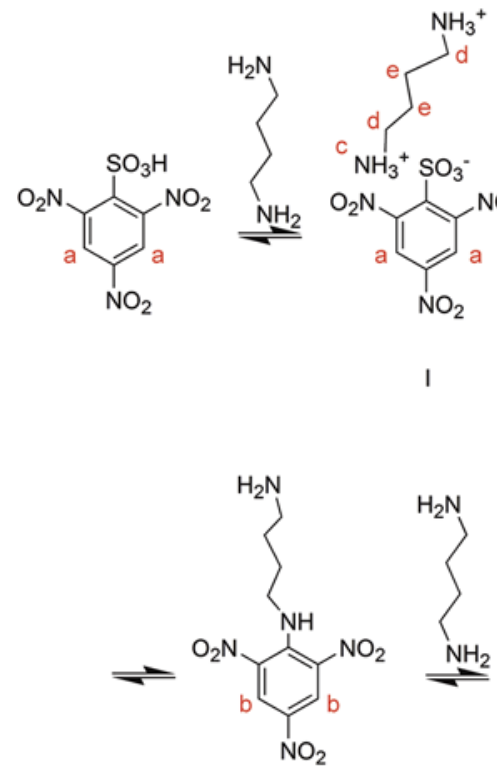

III
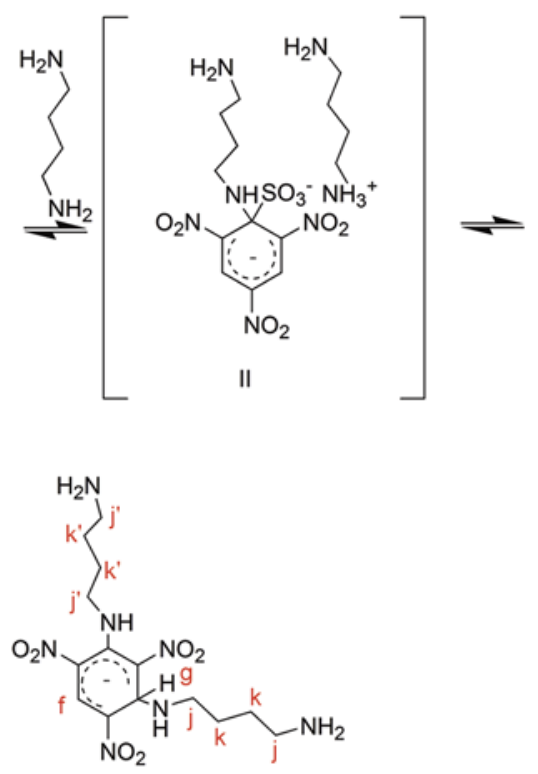

IV

Figure 6. Proposed mechanism of the reaction between TNBS and 1,4-diaminobutane (DAB) in DMSO 


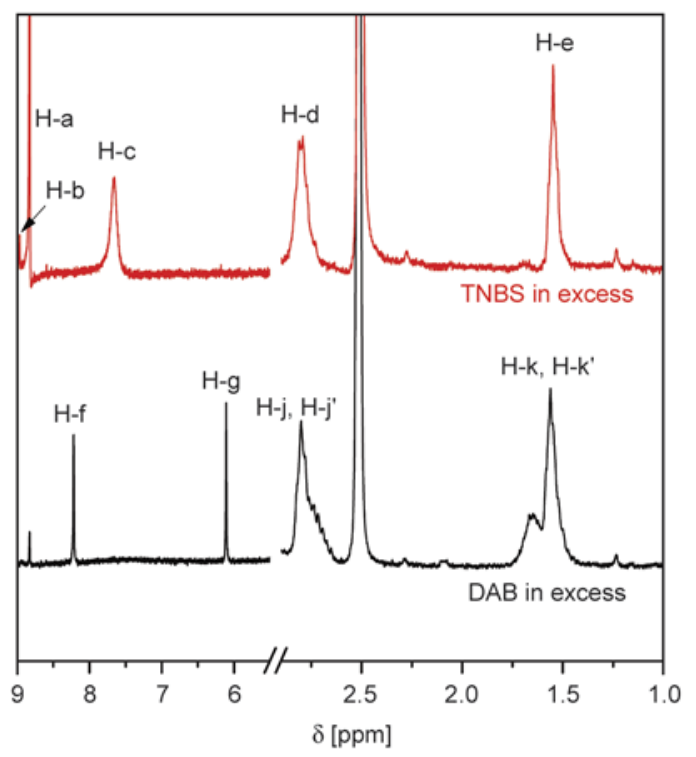

Figure 7. ${ }^{1} \mathrm{H}$ NMR spectra of the reaction product of TNBS and 1,4-diaminobutane in DMSO

In a large excess of DAB, the NMR spectrum is essentially different. The peak of TNBS $(\mathrm{H}-\mathrm{a})$ was reduced, indicating that only a small amount of the reagent remained in the mixture. The peak of ammonium group $(\mathrm{H}-\mathrm{c})$ disappeared; thus, we can assume that the equilibrium shifted towards the nucleophilic substitution of sulphonic acid groups and the formation of the aniline derivative. However, the peak of the H-b nucleus was not observed, whereas new peaks appeared at 8.1 and $6.0 \mathrm{ppm}$, indicating the formation of a new reaction product. The strong decrease in the chemical shift suggests the presence of an electron-rich chemical environment. The nucleophilic addition of primary amine groups to the unsubstituted ring positions resulted in the forma-

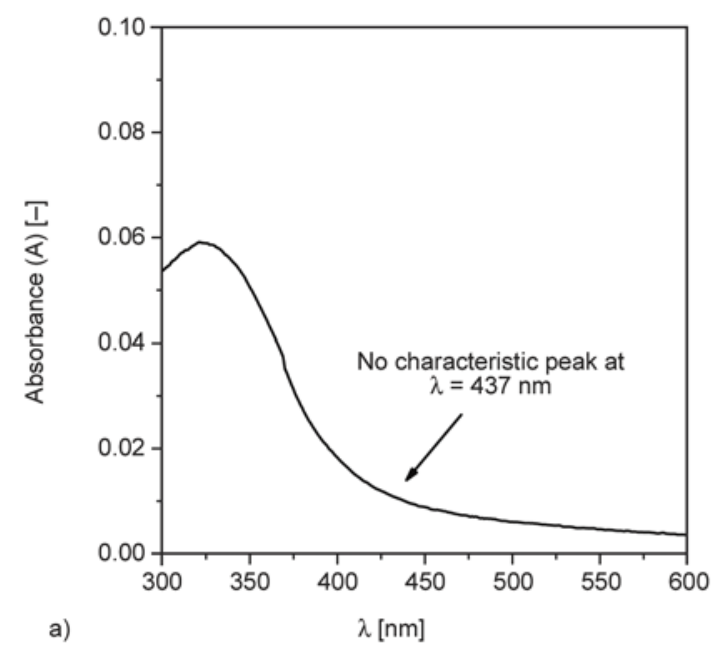

tion of a $\sigma$-complex (Figure $6, \mathrm{IV}$ ), which fits the chemical shifts ( 8.1 and $6.0 \mathrm{ppm})$ in the NMR spectrum [20]. The presence of this $\sigma$-complex is confirmed by the 1:1 intensity integrals of $\mathrm{H}-\mathrm{f}$ and $\mathrm{H}-\mathrm{g}$ nuclei.

The results prove that different species were present simultaneously in the reaction mixture. However, a reliable analysis requires a large excess of one of the reagents to shift the equilibrium to a well-defined product. Consequently, the molar ratio of TNBS to DAB was kept above 5 during the analysis of unreacted DAB to yield only the product I (Figure 6). Similarly, DAB was used in a 4-fold excess to TNBS during the quantitative determination of unreacted TNBS to yield only product IV (Figure 6).

\subsection{Determination of degree of chemical cross-linking of PSI-DAB gels in DMSO}

At first, unreacted cross-linker molecules were extracted from the PSI-DAB gel by washing with DMSO and the DAB molecules in the washing solution were reacted with TNBS. Similarly to the results obtained in aqueous medium, a characteristic peak $(\lambda=437 \mathrm{~nm})$ was not obtained in the absorption spectrum of the solution (Figure 8a), indicating that less than $0.1 \%$ of the cross-linker molecules remain unreacted after the cross-linking process, independently of the cross-linking ratio.

Secondly, the PSI-DAB gel was immersed in the solution of TNBS. The supernatant remained colourless indicating that the formation of picric acid was excluded in DMSO. Unreacted TNBS was extracted from the gel by washing with DMSO, and its concentration was determined from the absorbance

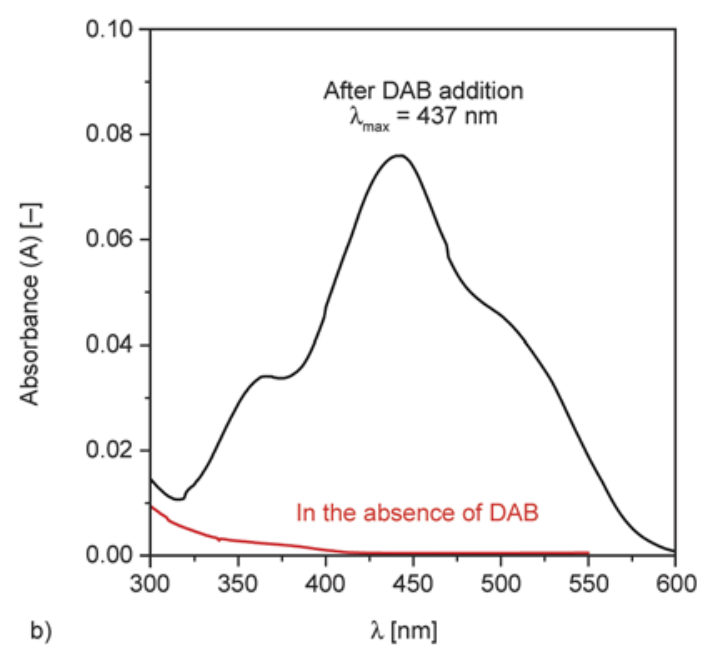

Figure 8. a) Absorption spectrum of the extracted washing solution of PSI-DAB gel after the addition of TNBS in DMSO; b) absorption spectrum of TNBS extracted from the gels in DMSO with and without DAB 
spectrum after the addition of DAB (Figure $8 \mathrm{~b}$ ). The reaction time was varied between 1 to 5 days, but it did not affect the measured amount of unreacted TNBS; thus, a reaction time of 1 day was applied in further experiments. The conversion rate of diamine molecules to cross-linking points was smaller than $100 \%$ in each case (Figure 9a). Consequently, the degree of chemical cross-linking was smaller than its theoretical maximum value calculated from the total conversion of cross-linker molecules (Figure 9b). The difference between the degree of chemical crosslinking and its theoretical maximum is rather small, but we observed a clear tendency between the conversion rate and the cross-linking ratio (Figure 9a). A larger cross-linking ratio results in a larger conversion rate, which contradicts the original expectation. However, the observed correlation can be explained by the development of a network structure during the cross-linking process. The amine groups of the crosslinker molecules and succinimide repeating units exhibit high reactivity; thus, a slightly cross-linked structure with pendant amine groups must form during the first stage of the cross-linking process. The presence of chemical cross-linking points reduces the segmental mobility of the polymer, which hinders the further formation of amide linkages; therefore, the structure formed in the initial stage of the process essentially affects any further reaction. In the case of a large cross-linking ratio, the polymer chains are densely cross-linked, which has a dual effect. First, the degree of swelling is significantly smaller than that of a less densely cross-linked gel (see the degree of swelling values in DMSO in Table 1)

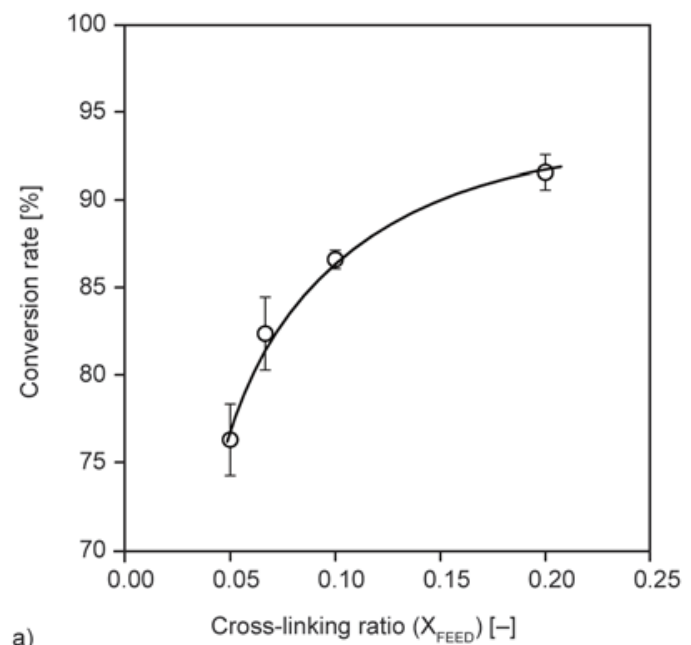

because of the elastic effect of net points, and the increased polymer concentration inside the forming gel accelerates the reaction. Second, the densely cross-linked structure orients the pendant amine groups for amide formation with the succinimide groups of adjacent polymer chains. Amide formation is supported by favourable distance of polymer chains and the orientation effect might be further strengthened by hydrogen bonds between nearby pendant amine groups. Further effects must be also important in this case e.g., electrostatic interactions in the polyelectrolyte network. All of these effects are less significant in a loosely cross-linked network, resulting in a lower conversion rate of cross-linker molecules. Nevertheless, the conversion rate was remarkably high $(>70 \%)$, and the degree of chemical cross-linking was also relatively high over the investigated range.

\subsection{Stability and accuracy}

TNBS was incubated in PVA gels for 3 days in the absence of free amines and approximately $99.9 \%$ of the feed amount of the reagent was recovered after careful washing of the gel. Therefore, the reagent is chemically stable in DMSO, and long-term measurements can be carried out without the formation of interfering by-products.

The accuracy of the TNBS assay was verified on PASP-CYS gels by quantifying of cysteamine after cleavage of disulphide linkages inside the gels. The presence of thiol groups during the analysis interferes with the reaction of TNBS and free amines, thus sodium bromate was used to oxidize excess

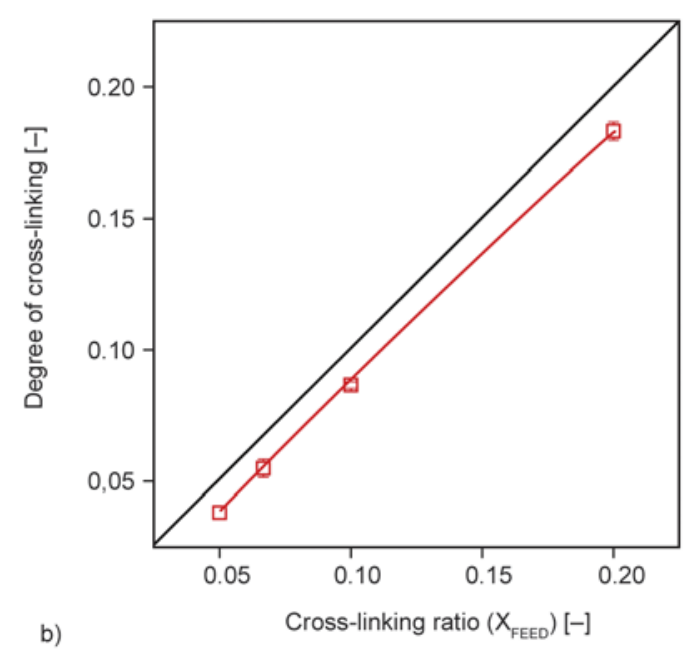

Figure 9. a) Conversion rate of diamine molecules to cross-linking points as a function of cross-linking ratio $\left(X_{\mathrm{FEED}}\right)$; b) Correlation between the degree of chemical cross-linking and the cross-linking ratio. The diagonal line represents $100 \%$ conversion. The curves on both diagrams are used to guide the eye. 


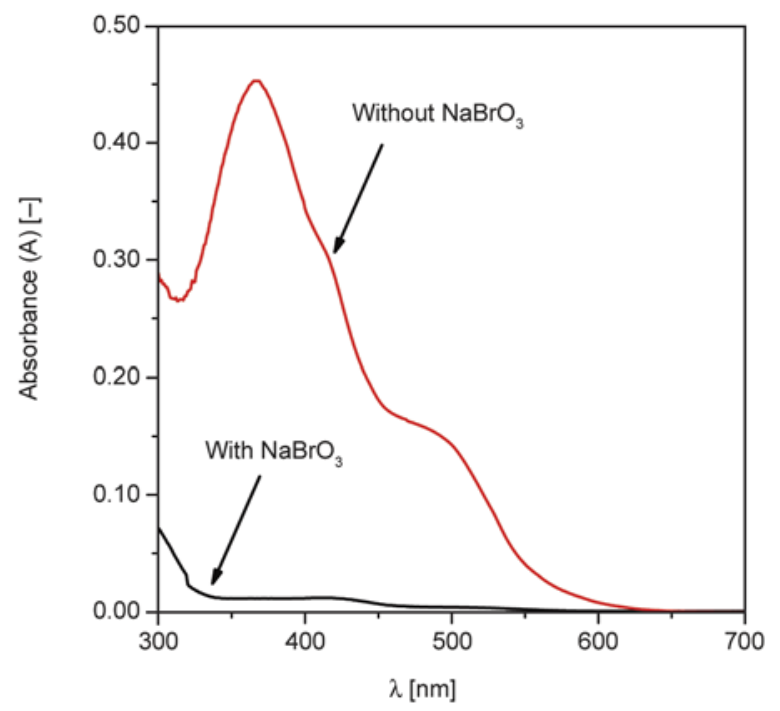

Figure 10. Absorption spectrum of TNBS in the presence of dithiothreitol (DTT) with and without the addition of sodium bromate $\left(\mathrm{NaBrO}_{3}\right)$ as oxidizing agent

DTT after dialysis (Figure 10). The number of pendant amine groups in the PSI-CYS gel was also determined in DMSO with the developed TNBS assay. Results show that $8.0 \pm 1.4 \%$ of cystamine do not form chemical cross-links, whereas the reduction-based method yielded a value of $11.3 \pm 1.8 \%$. According to an F-test, reproducibilities of the two methods are equal at a significance level of $95 \%$, and a paired t-test proved that the numbers of pendant amine groups obtained by the two methods are the same at a significance level of $95 \%$. Consequently, the method developed for the quantitative determination of pendant amine groups in DMSO exhibits satisfactory accuracy for chemically crosslinked aspartic acid-based polymer gels.

\section{Conclusions}

A novel method using 2,4,6-trinitrobenzensulphonic acid (TNBS) was developed to determine the degree of chemical cross-linking in aspartic acid-based polymer gels. The conventional TNBS method is reliable for the quantitative determination of small molecular amines in alkaline aqueous medium because the hydrolysis of the reagent does not interfere with the analysis if the reaction time is short. However, analysis of polymer networks bearing pendant amine groups is difficult in aqueous medium because of the formation of picric acid in a considerable amount. The TNBS assay was implemented in DMSO, and the reaction products were examined by ${ }^{1} \mathrm{H}$ NMR. The formation of picric acid was excluded, and the degree of chemical cross-linking of polysuccinimide gels was determined. Less than $0.1 \%$ of the cross-linker molecules remained unreacted because of the high reactivity of diamine molecules and the repeating units of the polymer. The conversion rate of diamine molecules to chemical cross-linking points was high but strongly depended on the feed composition of the gels. The stability of the reagent and accuracy of the developed method are satisfactory; thus, the method can be used to determine the degree of chemical crosslinking of PSI gels. Furthermore, this method can be extended to analyse other DMSO-swollen polymer gels bearing pendant amine groups.

\section{Acknowledgements}

This research was supported by the OTKA Foundation (PD76401) and by the New Széchenyi Development Plan (TÁMOP-4.2.1/B-09/1/KMR-2010-0002). András Szilágyi thanks the János Bolyai Research Scholarship of Hungarian Academy of Sciences for its support. The authors would like to thank Viktor Csókai for his help in the evaluation of the NMR spectra.

\section{References}

[1] Tanaka Y., Gong J. P., Osada Y.: Novel hydrogels with excellent mechanical performance. Progress in Polymer Science, 30, 1-9 (2005).

DOI: $10.1016 / j . p r o g p o l y m s c i .2004 .11 .003$

[2] Gyenes T., Torma V., Gyarmati B., Zrínyi M.: Synthesis and swelling properties of novel $\mathrm{pH}$-sensitive poly (aspartic acid) gels. Acta Biomaterialia, 4, 733-744 (2008).

DOI: 10.1016/j.actbio.2007.12.004

[3] Kopeček J.: Hydrogel biomaterials: A smart future? Biomaterials, 28, 5185-5192 (2007). DOI: 10.1016/j.biomaterials.2007.07.044

[4] Wang H., Shi Y., Wang L., Yang Z.: Recombinant proteins as cross-linkers for hydrogelations. Chemical Society Reviews, 42, 891-901 (2013). DOI: $10.1039 / \mathrm{c} 2 \mathrm{cs} 35358 \mathrm{j}$

[5] Çaykara T., Akçakaya I.: Swelling behaviors of ionic $\operatorname{poly}(N, N$-dimethylacrylamide-co-acrylamide) hydrogels in various media. Journal of Applied Polymer Science, 104, 2140-2145 (2007).

DOI: $10.1002 / a p p .24313$

[6] Xu K., Tan Y., Chen Q., An H., Li W., Dong L., Wang P.: A novel multi-responsive polyampholyte composite hydrogel with excellent mechanical strength and rapid shrinking rate. Journal of Colloid and Interface Science, 345, 360-368 (2010). DOI: $10.1016 /$ j.jcis.2010.01.058 
[7] Kersey F. R., Loveless D. M., Craig S. L.: A hybrid polymer gel with controlled rates of cross-link rupture and self-repair. Journal of the Royal Society Interface, 4, 373-380 (2007). DOI: $10.1098 /$ rsif.2006.0187

[8] De S. K., Aluru N. R., Johnson B., Crone W. C., Beebe D. J., Moore J.: Equilibrium swelling and kinetics of pH-responsive hydrogels: Models, experiments, and simulations. Journal of Microelectromechanical Systems, 11, 544-555 (2002). DOI: $10.1109 /$ jmems.2002.803281

[9] Flory P. J.: Principles of polymer chemistry. Cornell University Press, Ithaka (1953).

[10] Wagermaier W., Kratz K., Heuchel M., Lendlein A.: Characterization methods for shape-memory polymers. Advances in Polymer Science, 226, 97-145 (2010). DOI: $10.1007 / 12 \_200925$

[11] Treloar L. R. G.: The physics of rubber elasticity. Oxford University Press, Oxford (1958).

[12] Mark J. E., Erman B.: Rubberlike elasticity. Cambridge University Press, Cambridge (2007).

[13] Yang J., Wang F., Tan T.: Degradation behavior of hydrogel based on crosslinked poly(aspartic acid). Journal of Applied Polymer Science, 117, 178-185 (2010). DOI: 10.1002/app.31943

[14] Torma V., Gyenes T., Szakács Z., Zrínyi M.: A novel potentiometric method for the determination of real crosslinking ratio of poly(aspartic acid) gels. Acta Biomaterialia, 6, 1186-1190 (2010). DOI: $10.1016 /$ j.actbio.2009.08.045

[15] Kiranas E. R., Tzouwara Karayanni S. M., Karayannis M. I.: The reaction of glutamic acid and trinitrobenzenesulfonic acid-kinetic study and analytical application. Talanta, 44, 1113-1121 (1997).

DOI: 10.1016/s0039-9140(96)02205-9

[16] Cayot P., Tainturier G.: The quantification of protein amino groups by the trinitrobenzenesulfonic acid method: A reexamination. Analytical Biochemistry, 249, 184-200 (1997).

DOI: 10.1006/abio.1997.2161

[17] Brown H. H.: A study of 2,4,6-trinitrobenzenesulfonic acid for automated amino acid chromatography. Clinical Chemistry, 14, 967-978 (1968).

[18] Snyder S. L., Sobocisnki P. Z.: An improved 2,4,6-trinitrobenzenesulfonic acid method for the determination of amines. Analytical Biochemistry, 64, 284-288 (1975). DOI: 10.1016/0003-2697(75)90431-5

[19] Grotzky A., Manaka Y., Fornera S., Willeke M., Walde P.: Quantification of $\alpha$-polylysine: A comparison of four UV/Vis spectrophotometric methods. Analytical Methods, 2, 1448-1455 (2010). DOI: $10.1039 / \mathrm{c} 0 \mathrm{ay} 00116 \mathrm{c}$

[20] Crampton M. R.: The stabilities of Meisenheimer complexes. Part 15. The interactions of 2,4,6-trinitrobenzenesulphonate ions with sodium sulphite and with sodium hydroxide in water. Journal of the Chemical Society, Perkin Transactions 2, 1978, 343-346 (1978). DOI: $10.1039 / \mathrm{p} 29780000343$
[21] Ives D. J. G., Moseley P. G. N.: Picric acid in aqueous solution. Journal of the Chemical Society B: Physical Organic, 757-761 (1966).

DOI: $10.1039 / \mathrm{J} 29660000757$

[22] Means G. E., Congdon W. I., Bender M. L.: Reactions of 2,4,6-trinitrobenzenesulfonate ion with amines and hydroxide ion. Biochemistry, 11, 3564-3571 (1972). DOI: $10.1021 / \mathrm{bi00769a011}$

[23] Brannon-Peppas L., Peppas N. A.: Equilibrium swelling behavior of dilute ionic hydrogels in electrolytic solutions. Journal of Controlled Release, 16, 319-329 (1991). DOI: 10.1016/0168-3659(91)90009-3

[24] Kirchmajer D. M., Watson C. A., Ranson M., in het Panhuis M.: Gelapin, a degradable genipin cross-linked gelatin hydrogel. RSC Advances, 3, 1073-1081 (2013). DOI: $10.1039 / \mathrm{c} 2 \mathrm{ra} 22859 \mathrm{a}$

[25] Bubnis W. A., Ofner III C. M.: The determination of $\varepsilon-$ amino groups in soluble and poorly soluble proteinaceous materials by a spectrophotometrie method using trinitrobenzenesulfonic acid. Analytical Biochemistry, 207, 129-133 (1992).

DOI: 10.1016/0003-2697(92)90513-7

[26] Ofner III C. M., Bubnis W. A.: Chemical and swelling evaluations of amino group crosslinking in gelatin and modified gelatin matrices. Pharmaceutical Research, 13, 1821-1827 (1996). DOI: 10.1023/A:1016029023910

[27] Kuijpers A. J., Engbers G. H. M., Feijen J., De Smedt S. C., Meyvis T. K. L., Demeester J., Krijgsveld J., Zaat S. A. J., Dankert J.: Characterization of the network structure of carbodiimide cross-linked gelatin gels. Macromolecules, 32, 3325-3333 (1999).

DOI: $10.1021 / \mathrm{ma} 981929 \mathrm{v}$

[28] Vichasilp C., Nakagawa K., Sookwong P., Higuchi O., Kimura F., Miyazawa T.: A novel gelatin crosslinking method retards release of mulberry 1-deoxynojirimycin providing a prolonged hypoglycaemic effect. Food Chemistry, 134, 1823-1830 (2012). DOI: $10.1016 /$ j.foodchem.2012.03.086

[29] Gyarmati B., Vajna B., Némethy Á., László K., Szilágyi A.: Redox- and pH-responsive cysteamine-modified poly(aspartic acid) showing a reversible sol-gel transition. Macromolecular Bioscience, 13, 633-640 (2013). DOI: 10.1002/mabi.201200420

[30] Crampton M. R., Routledge P. J.: The stabilities of Meisenheimer complexes. Part 34. Kinetic studies of $\sigma$ adduct formation and nucleophilic substitution in the reactions of 2,4,6-trinitrophenetole with aliphatic amines in dimethyl sulphoxide. Journal of the Chemical Society, Perkin Transactions 2, 1984, 573-581 (1984). DOI: 10.1039/p29840000573 